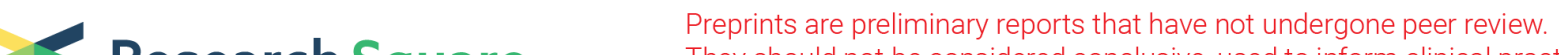 Research Square They should not be considered conclusive, used to inform clinical practice, or referenced by the media as validated information.
}

\section{The Lymph Node Ratio Optimizes Staging System while Adjuvant Chemotherapy Plays an Invalid Role for Patients with Nonmetastatic Colorectal Neuroendocrine Carcinomas: A Propensity Score Matching Analysis of Patients from the Surveillance, Epidemiology, and End- Results Database}

\section{Xiaoxiao Chen}

Jinhua Hospital of Zhejiang University: Jinhua Municipal Central Hospital

Hongjuan Zheng

Jinhua Hospital of Zhejiang University: Jinhua Municipal Central Hospital

Xia Zhang

Jinhua Hospital of Zhejiang University: Jinhua Municipal Central Hospital

Wanfen Tang

Jinhua Hospital of Zhejiang University: Jinhua Municipal Central Hospital

Shishi Zhou

Jinhua Hospital of Zhejiang University: Jinhua Municipal Central Hospital

\section{Xifeng Xu}

Jinhua Hospital of Zhejiang University: Jinhua Municipal Central Hospital

Jianfei Fu

Jinhua Hospital of Zhejiang University: Jinhua Municipal Central Hospital

Qinghua Wang ( $\square$ wqhw1001@zju.edu.cn)

Jinhua Hospital of Zhejiang University: Jinhua Municipal Central Hospital

\section{Research Article}

Keywords: Adjuvant chemotherapy, Colorectal Neuroendocrine Carcinomas, SEER, Age, Lymph node ratio

Posted Date: July 16th, 2021

DOl: https://doi.org/10.21203/rs.3.rs-682298/v1

License: (c) (7) This work is licensed under a Creative Commons Attribution 4.0 International License. Read Full License 


\section{Abstract}

Objective: Colorectal neuroendocrine carcinoma (CRNEC) is rare and little is known about survival benefit between lymph node ratio (LNR) and improved overall survival (OS), and so is the adjuvant chemotherapy (AC). We aim to evaluate the survival benefit of LNR and AC in patients with nonmetastatic CRNEC following resection.

Methods: Patients with resected nonmetastatic CRNECs were identified in Surveillance, Epidemiology, and End Results (SEER) during year 1992 to 2016. A Log-rank test was conducted to determine the survival difference. The survival benefit was evaluated using a competing-risks regression model and propensity score-matched (PSM) techniques were used to reduce the selection bias.

Results: A total of 251 patients met the inclusion criteria, of which, 152 patients $(60.56 \%)$ received AC. The age of $60(P=0.848)$ and number of 12 of resected regional lymph nodes $(P=0.082)$ acted as an optimal cutoff value in terms of survival, failing to reach a significance. Chemotherapy failed to bring survival benefit (hazard ratio [HR], $0.959 ; 95 \%$ confidence interval [CI], 0.649-1.416; $P=0.832$ ). Current $\mathrm{N}$ classification was not a significant predictor of patient survival (N1: $P=0.174$; N2: $P=0.028$, compared to N0, respectively). Multivariate analyses explored the revised $\mathrm{Nr}$ classification, based on LNR of 0.30 and 0.75 as cutoff value (Nr0: LNR£0.30; Nr1: 0.3<LNR£0.75; Nr2: LNR $>0.75$ ), as an independent prognostic factor ( $\mathrm{Nr} 1: P=0.003$; $\mathrm{Nr} 2: P<0.001$, compared to $\mathrm{Nr0}$, respectively). With the foundation of revised $\mathrm{Nr}$ classification, a revised TNrM was proposed for nonmetastatic CRNEC: stage I (T1-2Nr0), stage II (T1-2Nr1 or T3Nr0-1 or T4Nr0), and stage III (TxNr2 or T4Nr1). TNrM stage had better stratification according to Kaplan-Meier survival curves $(P<0.001)$.

Conclusions: AC seems invalid for improving the survival of patients with nonmetastatic CRNECs following resection. The LNR more accurately predict survival of CRNEC patients than current $\mathrm{N}$ classification.

\section{Background}

Neuroendocrine carcinomas (NECs) are frequent in the lung but rare in the gastrointestinal (GI) tract[1] while colorectal neuroendocrine carcinoma (CRNEC) is an even rare type of malignancy of extremely negative prognosis[2]. The poorly differentiated NECs are distinguished into small or large cells carcinoma according to 2010 WHO classification[3, 4].

Due to the rarity of CRNEC and the resulting difficulty to accumulate a large cohort for evaluation, there are few retrospective studies and almost no prospective studies to evaluate its prognostic value of adjuvant chemotherapy (AC)[2], resulting in the fact that most of the suggested guidelines is consulted to that of SCLC[1]. In clinical practice, relevant features addressing the optimal management strategy of NECs consists of clinical behavior, mitoses, Ki-67 expression, morphology and so on[5], but no standard clinical management are recommended to date according to the tumor location of origin[6]. It is suggested that "most clinicians would advocate platinum-based adjuvant therapy" after curative surgery, similar to the treatment guidelines for small cell lung cancer[2], which is considered as the current consensus from the European Neuroendocrine Tumor Society (ENETS)[7]. This is also accepted by guidelines of Neuroendocrine Tumor Society (NANETS) as well as National Comprehensive Cancer Network (NCCN) [8]. However, evidence supporting current guidelines are extremely limited, with the lack of large studies reported to improve survival associated with adjuvant chemotherapy for resectable CRNECs following resection. Rui Mao, et al[8] stated that patients with nonmetastatic CRNECs may benefit from AC, knowing that they included carcinoid tumor (8240), argentaffin 
carcinoid tumor (8241), enterochromaffin cell tumor (8242) et al with grade codes 3 (poorly differentiated) or 4 (undifferentiated; anaplastic) as NECs. According to 2010 WHO classification, the including criteria[9] that the International Classification of Diseases for Oncology codes for small cell neuroendocrine carcinoma and large cell neuroendocrine carcinoma is more precise for the diagnosis and meaningful for further analysis.

On the other hand, the Tumor, Lymph node, Metastases classification (TNM) is currently proposed as a dynamic and the most powerful prognostic instrument to tailor optimal prediction of cancer specific survival (CSS)[10]. Significantly, TNM staging system in colorectal neuroendocrine carcinoma is referred to that of colorectal adenocarcinoma, despite of the few studies assessing the value of current TNM classification for NECs[10]. Lymph node (LN) metastasis was demonstrated as a negative factor in small intestinal neuroendocrine tumors, and the lymph node ratio optimized the staging[1]. However, neither large-scale retrospective studies nor prospective studies have well demonstrated the prognostic value of lymph node ratio (LNR) in patients with nonmetastatic CRNECs. Therefore, by using a large-scale database, we performed the study to evaluate the survival benefit of AC and the current AJCC TNM staging system of CRNECs to further propose revised TNM staging systems based on the revised $\mathrm{N}$ classification.

\section{Methods}

\section{Study population}

A total of 251 patients with Colorectal Neuroendocrine Carcinoma (CR-NEC) were enrolled from The Surveillance, Epidemiology, and End Results (SEER) database, which was established by the National Cancer Institute with the aim of collecting information about cancer incidence and survival. The current SEER 18 database collects and publishes cancer data including cancer incidence and survival from 18 population-based cancer registries among 14 states across the United States, about $26 \%$ of the United States population (http://seer.cancer.gov/). The SEER*Stat software version 8.3.6 (http://seer.cancer.gov/seerstat) was used to identify patients microscopically confirmed as Colorectal Neuroendocrine Carcinoma from January 1992 to December 2016. Patients diagnosed after 2016 were excluded to ensure an adequate duration of follow-up. We obtained permission to access the research data (Reference Number: 12005-Nov2019). This study was approved by the review board of XXX. We retrieved records of year and age at diagnosis, gender, race, marital status, histological type, differentiated grade, tumor location, TNM staging, Chemotherapy, insurance status, CEA, cause of death and survival time from the SEER database.

The specific inclusion criteria were as follows: (1) site record ICD-0-3 was limited to colon and rectum (C18, C19, C20); (2) histological type ICD-0-3 was limited to large cell neuroendocrine carcinoma, small cell carcinoma and neuroendocrine carcinoma $(8013 / 3,8041 / 3,8246 / 3)$; (3) year of diagnosis was limited from 1992 to 2016; (4) the age at diagnosis was limited from 20 to 80; (5) patients were not multiple primary tumors.

The exclusion criteria were as follows: (1) patients were lacking documentation of race, sex, tumor location, number of resected regional lymph nodes or marital status; (2) the grade of tumor was unknown or G1 or G2; (3) patients who survived less than one month; (4) patients did not receive radical surgery.

Figure 1. The inclusion and exclusion criteria in SEER.

\section{Variable Declaration}


Age at diagnosis was regrouped as $<60$ years old and $\geq 60$ years old. Gender was classified as male and female. Marital status was grouped as married, single or divorced. Race was divided into white, black and others.

Differentiated grades were grouped as poorly differentiated and undifferentiated. Histology was grouped as large cell neuroendocrine carcinoma, small cell carcinoma and neuroendocrine carcinoma. Tumor location was grouped as right-side colon, left-side colon and rectum. Chemotherapy was divided into "yes" and "no". Insurance status was classified as insured, any Medicaid, uninsured and unknown.

\section{Statistical Analyses}

The distribution of clinicopathological characteristic in different groups was analyzed using Chi-Squared tests. The restrictive cubic spline graph (RCS) was used to determine reasonable cutoff values of regional lymph nodes and lymph node ratio. Propensity score matching method (PSM) was used to balance differences of characteristics between groups. The propensity score was calculated by logistic regression. The "Matchit" package in $\mathrm{R}$ software was used as the nearest method with ratio 1:1 and caliper $=0.01$. The cancer-specific survival (CSS), the primary endpoint, was calculated from the date of diagnosis to the date of death of tumor. Death attributed to other causes was defined as a censored observation. Survival curves were generated using the Kaplan-Meier method, and the log-rank test was carried out to evaluate the differences between groups. The 1year, 3-year and 5-year CSS rates were calculated by Life-Table method. The hazard ratio (HR) of the variables for CSS was estimated using Cox proportional hazard regression model. And univariate and multivariate analysis was also conducted using the Cox proportional hazards regression model with backward, stepwise elimination of variables. The cumulative incidence of cancer-specific death (CSD) and non-CSD were calculated by the Fine and Gray model.

The differences were considered statistically significant when the two-sided $P$-value was less than 0.05. R3.6.3 software (http://www.r-project.org/) was used to perform the statistical analyses.

\section{Results}

\section{Clinicopathological characteristics of matched patients with Colorectal Neuroendocrine Carcinoma}

We identified 251 eligible patients with Colorectal Neuroendocrine Carcinoma from SEER, and the average age of them was 62.8 (standard deviation SD, \pm 11.0) years old. Median follow-up time was 22.0 months (range, 1.0-234 months). After PSM according to age at diagnosis, there were 105 patients in young group ( $<60$ years old) and old group ( $\geq 60$ years old), respectively. And there were 99 patients in chemotherapy group and observation group, respectively after PSM according to chemotherapy status. All covariates were well balanced between groups (all $p>0.05$ ). The detail information was indicated in Table 1. 
Table 1

Descriptive characteristics of 251 patients of Study Population within the SEER Medicare-linked database and Propensity Score-Matched patients

\begin{tabular}{|c|c|c|c|c|c|c|c|}
\hline \multirow[t]{2}{*}{ characteristic } & \multirow[t]{2}{*}{$\begin{array}{l}N(\%) \\
(n=251)\end{array}$} & \multicolumn{3}{|c|}{$\begin{array}{l}\text { Propensity score-matched cohort } \\
(n=210)\end{array}$} & \multicolumn{3}{|c|}{$\begin{array}{l}\text { Propensity score-matched cohort } \\
(n=198)\end{array}$} \\
\hline & & $\begin{array}{l}\text { Age }<= \\
60(N= \\
105)\end{array}$ & $\begin{array}{l}\text { Age }>60 \\
(N=105)\end{array}$ & $\begin{array}{l}\mathrm{P} \\
\text { Value }\end{array}$ & $\begin{array}{l}\text { Observation } \\
(\mathrm{N}=99)\end{array}$ & $\begin{array}{l}\text { chemotherapy } \\
(\mathrm{N}=99)\end{array}$ & $\begin{array}{l}\mathrm{P} \\
\text { Value }\end{array}$ \\
\hline Age(years) & & & & $N A$ & & & 0.366 \\
\hline$\nabla 60$ & 105(41.83) & - & - & & $30(30.30)$ & $36(36.3636)$ & \\
\hline$\geq 60$ & $146(58.17)$ & - & - & & 69(69.70) & 63(63.64) & \\
\hline Gender & & & & 0.890 & & & 0.776 \\
\hline Female & 121(48.21) & $51(48.57)$ & $50(47.62)$ & & $48(48.48)$ & $46(46.46)$ & \\
\hline Male & 130(51.79) & $54(51.43)$ & $55(52.38)$ & & $51(51.52)$ & $53(53.54)$ & \\
\hline Race & & & & 0.787 & & & 0.907 \\
\hline White & 215(85.66) & $88(83.81)$ & $91(86.67)$ & & $86(86.87)$ & $86(86.86)$ & \\
\hline Black & $22(8.76)$ & $12(11.43)$ & $9(8.57)$ & & $10(10.10)$ & $9(9.09)$ & \\
\hline Other & $14(5.58)$ & $5(4.76)$ & $5(4.76)$ & & $3(3.03)$ & $4(4.04)$ & \\
\hline Marital status & & & & 0.300 & & & 0.887 \\
\hline Married & $152(60.56)$ & $69(65.71)$ & $70(66.67)$ & & $60(60.61)$ & $57(57.58)$ & \\
\hline Single & $39(15.54)$ & $21(20.00)$ & 14(13.33) & & 13(13.13) & 13(13.13) & \\
\hline Divorced & $60(23.9)$ & $15(14.29)$ & $21(20.00)$ & & $26(26.26)$ & $29(29.29)$ & \\
\hline Grade & & & & 0.238 & & & 0.536 \\
\hline Poorly & $166(66.14)$ & $67(63.81)$ & $75(71.43)$ & & $71(71.72)$ & $67(67.68)$ & \\
\hline Undifferentiated & $85(33.86)$ & $38(36.19)$ & $30(28.57)$ & & $28(28.28)$ & $32(32.32)$ & \\
\hline Histology & & & & 0.874 & & & 0.573 \\
\hline Large cell & $40(15.94)$ & 19(18.10) & $18(17.14)$ & & $13(13.13)$ & $15(15.15)$ & \\
\hline Small cell & $29(11.55)$ & $12(11.42)$ & 10(9.53) & & $9(9.09)$ & 13(13.13) & \\
\hline $\begin{array}{l}\text { Neuroendocrine } \\
\text { carcinoma }\end{array}$ & $182(72.51)$ & $74(70.48)$ & 77(73.33) & & 77(77.78) & 71(71.72) & \\
\hline
\end{tabular}

a Staging based on the 8th AJCC system of the colorectal carcinoma. $\mathrm{P}<0.05$ : the differences were considered statistically significant.

AJCC: American Joint Committee on Cancer;

NA: Nonapplication 


\begin{tabular}{|c|c|c|c|c|c|c|c|}
\hline \multirow[t]{2}{*}{ characteristic } & \multirow[t]{2}{*}{$\begin{array}{l}N(\%) \\
(n=251)\end{array}$} & \multicolumn{3}{|c|}{$\begin{array}{l}\text { Propensity score-matched cohort } \\
(n=210)\end{array}$} & \multicolumn{3}{|c|}{$\begin{array}{l}\text { Propensity score-matched cohort } \\
(n=198)\end{array}$} \\
\hline & & $\begin{array}{l}\mathrm{Age}<= \\
60(\mathrm{~N}= \\
105)\end{array}$ & $\begin{array}{l}\text { Age }>60 \\
(N=105)\end{array}$ & Value & $\begin{array}{l}\text { Observation } \\
(\mathrm{N}=99)\end{array}$ & $\begin{array}{l}\text { chemotherapy } \\
(\mathrm{N}=99)\end{array}$ & $\begin{array}{l}\mathrm{P} \\
\text { Value }\end{array}$ \\
\hline \multicolumn{2}{|l|}{ T classification ${ }^{a}$} & & & 0.934 & & & 0.535 \\
\hline T1 & 15(5.98) & $5(4.76)$ & $3(2.86)$ & & $8(8.08)$ & $5(5.05)$ & \\
\hline $\mathrm{T} 2$ & 19(7.57) & $10(9.53)$ & $8(7.62)$ & & $7(7.07)$ & $9(9.09)$ & \\
\hline Т3 & $141(56.18)$ & $58(55.24)$ & $60(57.14)$ & & $57(57.58)$ & $53(53.54)$ & \\
\hline T4 & $54(21.51)$ & $23(21.90)$ & $24(22.86)$ & & 17(17.17) & $25(25.25)$ & \\
\hline Unknown & $22(8.76)$ & $9(8.57)$ & $10(9.52)$ & & $10(10.10)$ & $7(7.07)$ & \\
\hline \multicolumn{2}{|l|}{$\begin{array}{l}\text { N classification } \\
a\end{array}$} & & & 0.875 & & & 0.302 \\
\hline NO & $51(20.32)$ & 20(19.06) & $23(21.91)$ & & $29(29.29)$ & $20(20.20)$ & \\
\hline N1 & $100(39.84)$ & $45(42.86)$ & $43(40.95)$ & & $38(38.38)$ & $40(40.40)$ & \\
\hline N2 & $100(39.84)$ & $40(38.10)$ & $39(37.14)$ & & $32(32.33)$ & $39(39.40)$ & \\
\hline \multicolumn{2}{|l|}{ Staging a } & & & 0.868 & & & 0.327 \\
\hline I & 13(5.18) & $4(3.81)$ & $5(4.76)$ & & $8(8.08)$ & $5(5.05)$ & \\
\hline II & $38(15.14)$ & 16(15.24) & 18(17.14) & & $21(21.21)$ & $15(15.15)$ & \\
\hline III & $200(79.68)$ & $85(80.95)$ & $82(78.10)$ & & $70(70.71)$ & 79(79.80) & \\
\hline \multicolumn{2}{|l|}{ Tumor site } & & & 0.757 & & & 0.584 \\
\hline Right side colon & $151(60.16)$ & $59(56.19)$ & $60(57.14)$ & & $65(65.66)$ & $65(65.66)$ & \\
\hline Left side colon & $52(20.72)$ & $21(20.00)$ & $24(22.86)$ & & $25(25.25)$ & $21(21.21)$ & \\
\hline Rectum & $48(19.12)$ & $25(23.81)$ & $21(20.00)$ & & $9(9.09)$ & $13(13.13)$ & \\
\hline \multicolumn{2}{|l|}{ Chemotherapy } & & & 0.186 & & & $N A$ \\
\hline NO & $99(39.44)$ & $30(28.57)$ & $39(37.14)$ & & - & - & \\
\hline YES & $152(60.56)$ & 75(71.43) & $66(62.86)$ & & - & - & \\
\hline \multicolumn{2}{|l|}{ Insurance } & & & 0.109 & & & 0.455 \\
\hline Insured & $136(54.18)$ & $53(50.48)$ & $55(52.38)$ & & $57(57.58)$ & $51(51.52)$ & \\
\hline \multicolumn{8}{|c|}{$\begin{array}{l}\text { a Staging based on the 8th AJCC system of the colorectal carcinoma. } P<0.05 \text { : the differences were } \\
\text { considered statistically significant. }\end{array}$} \\
\hline \multicolumn{8}{|c|}{ AJCC: American Joint Committee on Cancer; } \\
\hline \multicolumn{8}{|c|}{ NA: Nonapplication } \\
\hline
\end{tabular}




\begin{tabular}{|c|c|c|c|c|c|c|c|}
\hline \multirow[t]{2}{*}{ characteristic } & \multirow[t]{2}{*}{$\begin{array}{l}N(\%) \\
(n=251)\end{array}$} & \multicolumn{3}{|c|}{$\begin{array}{l}\text { Propensity score-matched cohort } \\
(n=210)\end{array}$} & \multicolumn{3}{|c|}{$\begin{array}{l}\text { Propensity score-matched cohort } \\
(n=198)\end{array}$} \\
\hline & & $\begin{array}{l}\mathrm{Age}<= \\
60(\mathrm{~N}= \\
105)\end{array}$ & $\begin{array}{l}\text { Age }>60 \\
(N=105)\end{array}$ & $\begin{array}{l}\mathrm{P} \\
\text { Value }\end{array}$ & $\begin{array}{l}\text { Observation } \\
(\mathrm{N}=99)\end{array}$ & $\begin{array}{l}\text { chemotherapy } \\
(\mathrm{N}=99)\end{array}$ & $\stackrel{P}{\text { Value }}$ \\
\hline Any medicaid & $14(5.58)$ & $10(9.52)$ & $4(3.81)$ & & $8(8.08)$ & $5(5.05)$ & \\
\hline Uninsured & $6(2.39)$ & $5(4.76)$ & $1(0.95)$ & & $3(3.03)$ & $2(2.02)$ & \\
\hline Unknown & $95(37.85)$ & $37(35.24)$ & $45(42.86)$ & & $31(31.31)$ & $41(41.41)$ & \\
\hline
\end{tabular}

a Staging based on the 8th AJCC system of the colorectal carcinoma. $P<0.05$ : the differences were considered statistically significant.

AJCC: American Joint Committee on Cancer;

NA: Nonapplication

\section{Exploration of the impact on CSS of resected regional lymph nodes}

The end of follow-up time was November, 2016. The 1-, 3- and 5-year CSS rates for patients with resected regional lymph nodes $\geq 12(60.9 \%, 33.0 \%, 22.3 \%$ respectively) were higher than those with resected regional lymph nodes $<12(53.7 \%, 24.1 \%, 20.3 \%$ respectively) with $P=0.082$. With resected regional lymph nodes as a continuous variable, the HR of CSS for regional lymph nodes $<12$ decreased, but started to increase when regional lymph nodes $\geq 12$ (Fig. 2B). Therefore, a total of 12 was chosen as an optimal cutoff value of regional lymph nodes. CRNEC patients who with resected regional lymph nodes $\geq 12$ had similar CSS with the patients with resected regional lymph nodes $<12(P=0.082)$ (Fig. 2).

Figure 2 Impact of resected regional lymph nodes on CSS in CRNEC. A The overall cancer-specific survival (CSS) between in patients with CRNEC. B CSS between resected regional lymph nodes $<12$ and resected regional lymph nodes $\geq 12$ in the patients. C Restrict cubic spline for resected regional lymph nodes.

\section{Exploration of the impact on CSS of age at diagnosis}

The age of 60 was chosen as an optimal cutoff value according to the RCS method (Fig. 3A). After the univariate and multivariate analyses for age, we found that there was no direct relationship between age and CSS (Table 2). There was no statistically significant difference between younger group (age $\leq 60)$ and older group (age $>60)$ in the raw cohort for either CSD or CSS ( $p$ value, 0.879 and 0.848 , respectively) (Fig. 3B, 3C). Besides, the same phenomenon was proved in the PSM cohort with $p=0.939$ (Fig. 3D). 
Table 2

Univariate and Multivariate Analyses of Predictors of CRC-NEC In the Study Population

\begin{tabular}{|c|c|c|c|c|c|c|c|c|c|c|}
\hline \multirow{2}{*}{$\begin{array}{l}\text { Characteristic } \\
(n=344)\end{array}$} & \multirow{2}{*}{$\begin{array}{l}N \\
(251)\end{array}$} & \multirow{2}{*}{$\begin{array}{l}1- \\
\text { year } \\
\text { CSS }^{a}\end{array}$} & \multirow{2}{*}{$\begin{array}{l}\text { 3- } \\
\text { year } \\
\text { CSS }^{a}\end{array}$} & \multirow{2}{*}{$\begin{array}{l}5- \\
\text { year } \\
\text { CSS }^{a}\end{array}$} & \multicolumn{3}{|c|}{ Univariate analysis ${ }^{b}$} & \multicolumn{3}{|c|}{ Multivariate analysis ${ }^{\text {b }}$} \\
\hline & & & & & HR & $95 \% \mathrm{Cl}$ & $\begin{array}{l}P \\
\text { value }\end{array}$ & HR & $95 \% \mathrm{Cl}$ & $\begin{array}{l}\text { P } \\
\text { Value }\end{array}$ \\
\hline \multicolumn{11}{|l|}{ Age } \\
\hline$\nabla 60$ & 105 & 57.14 & 33.33 & 25.71 & 1 & & & 1 & & \\
\hline$\geq 60$ & 146 & 60.96 & 29.45 & 19.18 & 1.015 & $\begin{array}{l}0.730- \\
1.411\end{array}$ & 0.928 & 1.032 & $\begin{array}{l}0.689- \\
1.5459\end{array}$ & 0.879 \\
\hline \multicolumn{11}{|l|}{ Gender } \\
\hline Female & 121 & 65.29 & 24.79 & 13.30 & 1 & & & 1 & & \\
\hline Male & 130 & 53,85 & 28.46 & 19.23 & 1.148 & $\begin{array}{l}0.831- \\
1.586\end{array}$ & 0.403 & 1.127 & $\begin{array}{l}0.777- \\
1.636\end{array}$ & 0.528 \\
\hline \multicolumn{11}{|l|}{ Race } \\
\hline White & 215 & 59.53 & 30.23 & 20.93 & 1 & & & 1 & & \\
\hline Black & 22 & 50.00 & 36.36 & 22.73 & 1.109 & $\begin{array}{l}0.614- \\
2.003\end{array}$ & 0.731 & 1.387 & $\begin{array}{l}0.736- \\
2.615\end{array}$ & 0.311 \\
\hline Other & 14 & 71.43 & 35.71 & 28.57 & 0.924 & $\begin{array}{l}0.457- \\
1.868\end{array}$ & 0.826 & 0.830 & $\begin{array}{l}0.329- \\
2.096\end{array}$ & 0.693 \\
\hline \multicolumn{11}{|l|}{ Marital status } \\
\hline Married & 152 & 63.82 & 36.84 & 26.97 & 1 & & & 1 & & \\
\hline Single & 39 & 46.15 & 17.95 & 5.13 & 1.188 & $\begin{array}{l}0.738- \\
1.913\end{array}$ & 0.477 & 1.058 & $\begin{array}{l}0.620- \\
1.803\end{array}$ & 0.837 \\
\hline Divorced & 60 & 56.67 & 25.00 & 20.00 & 1.097 & $\begin{array}{l}0.756- \\
1.593\end{array}$ & 0.627 & 0.963 & $\begin{array}{l}0.616- \\
1.507\end{array}$ & 0.870 \\
\hline \multicolumn{11}{|l|}{ Grade } \\
\hline Poorly & 166 & 65.66 & 36.75 & 27.11 & 1 & & & 1 & & \\
\hline Undifferentiated & 85 & 47.06 & 20.00 & 11.76 & 1.479 & $\begin{array}{l}1.051- \\
2.080\end{array}$ & 0.025 & 1.585 & $\begin{array}{l}1.057- \\
2.377\end{array}$ & 0.026 \\
\hline \multicolumn{11}{|l|}{ Histology } \\
\hline Large cell & 40 & 52.50 & 10.00 & 10.00 & 1 & & & 1 & & \\
\hline Small cell & 29 & 55.17 & 24.14 & 17.24 & 1.251 & $\begin{array}{l}0.730- \\
2.146\end{array}$ & 0.415 & 1.138 & $\begin{array}{l}0.560- \\
2.314\end{array}$ & 0.721 \\
\hline
\end{tabular}

\footnotetext{
a Five-year overall survival rate was calculated using Kaplan-Meier methods; ${ }^{b}$ univariate and multivariate analyses were conducted using competing-risks regression model; ${ }^{\mathrm{C}}$ staging based on the 8th AJCC system of the colorectal carcinoma. AJCC, American Joint Committee on Cancer; CSS, cancer specific survival; HR, hazard ratio. $P<0.05$ : the differences were considered statistically significant.
} 


\begin{tabular}{|c|c|c|c|c|c|c|c|c|c|c|}
\hline \multirow{2}{*}{$\begin{array}{l}\text { Characteristic } \\
(n=344)\end{array}$} & \multirow{2}{*}{$\begin{array}{l}N \\
(251)\end{array}$} & \multirow{2}{*}{$\begin{array}{l}1- \\
\text { year } \\
\text { CSS }^{a}\end{array}$} & \multirow{2}{*}{$\begin{array}{l}\text { 3- } \\
\text { year } \\
\text { CSS }^{a}\end{array}$} & \multirow{2}{*}{$\begin{array}{l}\text { 5- } \\
\text { year } \\
\text { CSS }^{a}\end{array}$} & \multicolumn{3}{|c|}{ Univariate analysis ${ }^{b}$} & \multicolumn{3}{|c|}{ Multivariate analysis ${ }^{b}$} \\
\hline & & & & & HR & $95 \% \mathrm{Cl}$ & $\begin{array}{l}P \\
\text { value }\end{array}$ & HR & $95 \% \mathrm{Cl}$ & $\begin{array}{l}\mathrm{P} \\
\text { Value }\end{array}$ \\
\hline $\begin{array}{l}\text { Neuroendocrine } \\
\text { carcinoma }\end{array}$ & 182 & 61.54 & 34.62 & 25.27 & 0.836 & $\begin{array}{l}0.535- \\
1.306\end{array}$ & 0.432 & 0.988 & $\begin{array}{l}0.442- \\
2.205\end{array}$ & 0.976 \\
\hline \multicolumn{11}{|l|}{ T classification ${ }^{c}$} \\
\hline T1 & 15 & 86.67 & 40.00 & 33.33 & 1 & & & 1 & & \\
\hline T2 & 19 & 89.47 & 47.37 & 26.32 & 0.907 & $\begin{array}{l}0.341- \\
2.409\end{array}$ & 0.844 & 1.098 & $\begin{array}{l}0.397- \\
3.041\end{array}$ & 0.857 \\
\hline T3 & 141 & 65.96 & 36.88 & 24.82 & 1.518 & $\begin{array}{l}0.687- \\
3.353\end{array}$ & 0.302 & 1.504 & $\begin{array}{l}0.598- \\
3.784\end{array}$ & 0.386 \\
\hline $\mathrm{T} 4$ & 54 & 42.59 & 20.37 & 16.67 & 2.745 & $\begin{array}{l}1.189- \\
6.337\end{array}$ & 0.018 & 2.472 & $\begin{array}{l}1.000- \\
6.107\end{array}$ & 0.050 \\
\hline Unknown & 22 & 13.64 & 0.00 & 0.00 & 3.274 & $\begin{array}{l}1.270- \\
8.438\end{array}$ & 0.014 & 3.303 & $\begin{array}{l}1.167- \\
9.355\end{array}$ & 0.024 \\
\hline \multicolumn{11}{|l|}{$\begin{array}{l}\mathrm{N} \\
\text { classification }^{\mathrm{c}}\end{array}$} \\
\hline NO & 51 & 90.20 & 68.63 & 41.18 & 1 & & & 1 & & \\
\hline N1 & 100 & 63.00 & 26.00 & 22.00 & 3.910 & $\begin{array}{l}2.120- \\
7.211\end{array}$ & 0.000 & 2.242 & $\begin{array}{l}0.701- \\
7.169\end{array}$ & 0.174 \\
\hline N2 & 100 & 40.00 & 17.00 & 12.00 & 6.682 & $\begin{array}{l}3.640- \\
12.265\end{array}$ & 0.000 & 3.804 & $\begin{array}{l}1.151- \\
12.572\end{array}$ & 0.028 \\
\hline \multicolumn{11}{|l|}{ Staging ${ }^{a}$} \\
\hline I & 13 & 92.31 & 69.23 & 38.46 & 1 & & & & & \\
\hline II & 38 & 89.47 & 68.42 & 42.11 & 0.922 & $\begin{array}{l}0.264- \\
3.228\end{array}$ & 0.899 & - & - & - \\
\hline III & 200 & 51.50 & 21.50 & 17.00 & 4.766 & $\begin{array}{l}1.635- \\
13.892\end{array}$ & 0.004 & - & - & - \\
\hline \multicolumn{11}{|l|}{ Tumor site } \\
\hline Right side colon & 151 & 61.59 & 33.77 & 21.85 & 1 & & & 1 & & \\
\hline Left side colon & 52 & 48.08 & 25.00 & 21.15 & 1.483 & $\begin{array}{l}0.950- \\
2.315\end{array}$ & 0.083 & 1.614 & $\begin{array}{l}0.992- \\
2.625\end{array}$ & 0.054 \\
\hline Rectum & 48 & 64.58 & 33.33 & 20.83 & 1.148 & $\begin{array}{l}0.788- \\
1.673\end{array}$ & 0.471 & 0.922 & $\begin{array}{l}0.589- \\
1.444\end{array}$ & 0.722 \\
\hline
\end{tabular}

\footnotetext{
${ }^{a}$ Five-year overall survival rate was calculated using Kaplan-Meier methods; ${ }^{b}$ univariate and multivariate analyses were conducted using competing-risks regression model; ${ }^{\mathrm{c}}$ staging based on the 8th AJCC system of the colorectal carcinoma. AJCC, American Joint Committee on Cancer; CSS, cancer specific survival; HR, hazard ratio. $P<0.05$ : the differences were considered statistically significant.
} 


\begin{tabular}{|c|c|c|c|c|c|c|c|c|c|c|}
\hline \multirow{2}{*}{$\begin{array}{l}\text { Characteristic } \\
(n=344)\end{array}$} & \multirow{2}{*}{$\begin{array}{l}\mathrm{N} \\
(251)\end{array}$} & \multirow{2}{*}{$\begin{array}{l}1- \\
\text { year } \\
\text { CSS }^{a}\end{array}$} & \multirow{2}{*}{$\begin{array}{l}\text { 3- } \\
\text { year } \\
\text { CSS }^{a}\end{array}$} & \multirow{2}{*}{$\begin{array}{l}\text { 5- } \\
\text { year } \\
\text { CSS }^{a}\end{array}$} & \multicolumn{3}{|c|}{ Univariate analysis ${ }^{b}$} & \multicolumn{3}{|c|}{ Multivariate analysis $^{b}$} \\
\hline & & & & & $\mathrm{HR}$ & $95 \% \mathrm{Cl}$ & $\begin{array}{l}P \\
\text { value }\end{array}$ & $\mathrm{HR}$ & $95 \% \mathrm{Cl}$ & $\begin{array}{l}\mathrm{P} \\
\text { Value }\end{array}$ \\
\hline \multicolumn{11}{|l|}{ Chemotherapy } \\
\hline NO & 99 & 60.61 & 39.39 & 27.27 & 1 & & & 1 & & \\
\hline YES & 152 & 58.55 & 25.66 & 18.42 & 1.334 & $\begin{array}{l}0.936- \\
1.903\end{array}$ & 0.111 & 0.959 & $\begin{array}{l}0.649- \\
1.416\end{array}$ & 0.832 \\
\hline \multicolumn{11}{|l|}{ Insurance } \\
\hline Insured & 136 & 59.56 & 33.09 & 20.59 & 1 & & & 1 & & \\
\hline Any Medicaid & 14 & 35.71 & 14.29 & 7.14 & 1.237 & $\begin{array}{l}0.496- \\
3.083\end{array}$ & 0.649 & 0.630 & $\begin{array}{l}0.231- \\
1.715\end{array}$ & 0.366 \\
\hline Uninsured & 6 & 50.00 & 16.67 & 0.00 & 1.144 & $\begin{array}{l}0.378- \\
3.464\end{array}$ & 0.811 & 1.350 & $\begin{array}{l}0.386- \\
4.721\end{array}$ & 0.638 \\
\hline Unknown & 95 & 63.16 & 30.53 & 26.32 & 1.365 & $\begin{array}{l}0.982- \\
1.898\end{array}$ & 0.064 & 1.321 & $\begin{array}{l}0.874- \\
1.998\end{array}$ & 0.187 \\
\hline \multicolumn{11}{|c|}{$\begin{array}{l}{ }^{a} \text { Five-year overall survival rate was calculated using Kaplan-Meier methods; }{ }^{b} \text { univariate and multivariate } \\
\text { analyses were conducted using competing-risks regression model; }{ }^{c} \text { staging based on the } 8 \text { th AJCC system of } \\
\text { the colorectal carcinoma. AJCC, American Joint Committee on Cancer; CSS, cancer specific survival; HR, } \\
\text { hazard ratio. } P<0.05 \text { : the differences were considered statistically significant. }\end{array}$} \\
\hline
\end{tabular}

Figure 3 Impact of age at diagnosis on CSD and CSS in CR-NEC. A Restrict cubic spline for age at diagnosis. B The CSD and non-CSD between younger group (age $\leq 60)$ and older group (age $>60)$. C The CSS between younger group and older group in the raw cohort. D The CSS between younger group and older group in the PSM cohort.

\section{Exploration of the impact on CSS of chemotherapy after radical surgery}

The 1-, 2- and 5-year CSS rates for patients with chemotherapy (58.55\%, $25.66 \%$ and $18.42 \%$,respectively) were similar with those without chemotherapy $(60.61 \%, 39.39 \%$ and $27.27 \%$ respectively) with $\mathrm{P}=0.832$ (Table 2). The CSD and CSS between chemotherapy and non-chemotherapy groups in the raw cohort were no statistically significant difference ( $p$ value, 0.832 and 0.119 , respectively) (Fig. 4A, 4B). And in the PSM cohort, there was still no statistically significant difference between chemotherapy and non-chemotherapy groups with $p=0.259$ (Fig. 4C).

In the CR-NEC patients with stage I, patients who received chemotherapy had worse CSS than those did not received chemotherapy with $p=0.020$ (Fig. 5A). However, in stage II and stage III, patients with chemotherapy did not obtain better CSS than those without chemotherapy (Fig. 5B, 5C).

Figure 4 Impact of chemotherapy on CSD and CSS in CRNEC. A The CSD and non-CSD between chemotherapy and non-chemotherapy groups in the raw cohort. B The CSS between chemotherapy and non-chemotherapy groups in the raw cohort. C The CSS between chemotherapy and non-chemotherapy groups in the PSM cohort. 
Figure 5 Impact of chemotherapy on CSS in CRNEC patients with different stage. A The CSS between chemotherapy and non-chemotherapy groups in patients with stage I. B The CSS between chemotherapy and non-chemotherapy groups in patients with stage II. C The CSS between chemotherapy and non-chemotherapy groups in patients with stage III.

\section{The impact of different stages according to 8th AJCC TNM staging system on CSS}

According to 8th AJCC TNM staging system (Fig. 6A), Kaplan-Meier survival analysis showed that patients with stage III had worse CSS than stage I and stage II with statistically significant difference $(p<0.001)$, however, patients with stage I and stage II had similar CSS without statistic difference (Fig. 6B).

\section{The impact of Lymph node ratio (LNR) on CSS}

Lymph nodes ratio, the ratio of the number of positive lymph nodes to the number of resected regional lymph nodes, was a new concept that we proposed in this study. The 0.3 and 0.75 were chosen as optimal cutoff values of LNR according to the RCS method (Fig. 6C), then LNR was regrouped as NrO (LNR $\leq 0.3), N r 1(0.3<L N R \leq 0.75)$ and $\mathrm{Nr} 2(\mathrm{LNR}>0.75)$. And further Kaplan-Meier survival analysis according to different LNR groups verified the rationality of these two cutoff values. The results showed that $\mathrm{NrO}$ had a better CSS than Nr1, while Nr1 had a better CSS than Nr2, with statistically significant differences $(p=0.003)$ (Fig. 6D).

Figure 6A The current TNM staging system of AJCC-8. B The CSS among different stages in patients with CRNEC. C Restrict cubic spline for lymph nodes ratio (LNR). D The CSS among different LNR groups in patients with CR-NEC.

\section{Revised TNM staging system according to the combination of 8th AJCC TNM staging system and LNR.}

According to the combination of 8th AJCC TNM staging system and LNR, we rebuilt a new TNM staging system, and the detail information was indicated in Fig. 7A. Multivariate analyses showed that Nr1 and $\mathrm{Nr} 2 \mathrm{were}$ associated with a worse CSS with $p=0.003$ and $p<0.001$, respectively (Table 3 ). And the predictive value of the current TNM staging system in the patients with CRNEC was shown in Fig. 7B. The results revealed that patients with stage III had worse CSS than stage II, while patients stage II had worse CSS than those with stage I. The difference between them were statistically significant both with $p<0.001$. 
Table 3

Multivariate Analyses of Revised Nr classification

\begin{tabular}{|c|c|c|c|}
\hline \multirow[t]{2}{*}{ Characteristic } & \multicolumn{3}{|c|}{ Multivariate analysis ${ }^{a}$} \\
\hline & HR & $95 \% \mathrm{Cl}$ & $P$ value \\
\hline Age & 1.002 & $0.987-1.0187$ & 0.758 \\
\hline \multicolumn{4}{|l|}{ Grade } \\
\hline Poorly & 1 & & \\
\hline Undifferentiated & 1.475 & $1.044-2.086$ & 0.028 \\
\hline \multicolumn{4}{|l|}{ T classification ${ }^{b}$} \\
\hline $\mathrm{T} 1$ & 1 & & \\
\hline T2 & 0.857 & $0.321-2.286$ & 0.758 \\
\hline T3 & 1.181 & $0.535-2.608$ & 0.680 \\
\hline T4 & 2.278 & $0.992-5.235$ & 0.052 \\
\hline Unknown & 1.966 & $0.717-5.394$ & 0.189 \\
\hline \multicolumn{4}{|l|}{$\mathrm{Nr}$ classification ${ }^{b}$} \\
\hline $\mathrm{NrO}$ & 1 & & \\
\hline $\mathrm{Nr} 1$ & 1.839 & $1.231-2.747$ & 0.003 \\
\hline $\mathrm{Nr} 2$ & 2.824 & $1.768-4.509$ & 0.000 \\
\hline \multicolumn{4}{|l|}{ Tumor site } \\
\hline Right side colon & 1 & & \\
\hline Left side colon & 1.473 & $0.948-2.287$ & 0.085 \\
\hline Rectum & 1.049 & $0.680-1.619$ & 0.827 \\
\hline
\end{tabular}

Figure 7Revised TNM staging system and its prognostic capability A Revised TNM staging system according to the combination of 8th AJCC TNM staging system and lymph node ratio. B The CSS among different stages according to revised TNM staging system.

\section{Discussion}

Population-based analysis provide a fighting chance to improve our interpretations of this kind of rare malignancy due to the lack of prospective study in CRNECs. Using the SEER cancer registry, a total of 251 patients met the inclusion criteria, of which, 152 patients $(60.56 \%)$ received AC. With propensity score-matched 
techniques, the analysis gave a robust conclusion by reducing the selection bias and improving the validity. We demonstrated that AC was invalid for improved CSS in patients with nonmetastatic CRNECs regardless of tumor stages. To the best of our knowledge, it is the first study with more than 250 patients to provide evidence to reject AC for patients with resectable CRNECs. Of interest, patients with left-sided CRNECs may had a poorer prognosis compared to those with right-sided disease, although it failed to reach a statistic significance $(\mathrm{HR}, 1.614 ; 95 \% \mathrm{Cl}$, 0.992-2.235; $P=0.054$ ). In addition, our findings demonstrated that current $\mathrm{N}$ classification was not a significant predictor of patient survival. Multivariate analyses explored the revised Nr classification, based on LNR of 0.30 and 0.75 as cutoff value (NrO: LNR $\leq 0.30 ; \mathrm{Nr} 1: 0.3<\mathrm{LNR} \leq 0.75 ; \mathrm{Nr} 2$ : LNR > 0.75), as an independent prognostic factor. With the foundation of revised Nr classification, a revised TNrM was proposed for nonmetastatic CRNEC: stage I (T1-2Nr0), stage II (T1-2Nr1 or T3NrO-1 or T4NrO), and stage III (TxNr2 or T4Nr1). TNrM stage had better stratification according to Kaplan-Meier survival curves $(P<0.001)$.

In the present study, we found that the age of $60(P=0.848)$ and number of 12 of resected regional lymph nodes $(P=0.082)$ acted as an optimal cutoff value in terms of survival, failing to reach a statistic significance. It is well known that resection of more than 12 lymph nodes is associated with better prognosis in common colorectal carcinoma besides its pivotal role in accurate staging[11, 12], however it failed to reach a significance in CRNECs, which consisted of small cell neuroendocrine carcinoma and large cell neuroendocrine carcinoma. As an important indicator of treatment quality and therapeutic implications in early-stage patients, total LN yield in colon cancer resection specimens is influenced critically by extent of surgical resection and the following pathological evaluation[12], and a low LN yield in a resection specimen may indicate an inadequate surgical resection or unsatisfactory pathological examination[12,13]. Better survival may be achieved even in patients with positive LN in case of a higher number of recovered nodes[12]. Alternatively, a low LN yield may also be a reflection of a unique biological characteristics of the tumors such as the MSI phenotype, instead of the situation mentioned above[12]. Despite of the lack of a statistic significance, number of more than 12 of resected regional LN was associated with a better survival, showing in Fig. 3A, and further validation should be organized in the future in a larger sample of patients.

Incidence of NECs is rare, with a poor prognosis and commonly arising in the esophagus and large bowel[14], approximately 1000 cases annually[15]. As the most common site of extrapulmonary NECs with an increasing incidence, gastro-entero-pancreatic neuroendocrine carcinomas (GEP-NECs) represents less than $1 \%$ of all GI malignancies[1, 9, 16], with about $7 \%$ in the pancreas[1], and about to $40 \%$ in the colon[1, 8, 17]. Poorly differentiated neuroendocrine carcinomas, reflected by the number of mitoses or the Ki-67-positive fraction of > $20 \%[18]$, are distinguished into large or small cells in 2010 WHO classification[3, 4]. High-grade neuroendocrine carcinomas (HGNECs) are characterized by a high mitotic rate and a worse prognosis than common neuroendocrine tumors[19], and show neuro-endocrine marker expression, such as synaptophysin or chromogranin A[20]. Despite of an increase in the incidence over recent years, CRNECs is still a rare and aggressive type of malignancy of dismal prognosis[2, 8, 17], less than $1 \%$ of colorectal carcinomas[2, 7]. Many studies illustrated a poorer prognosis of colorectal compared to pancreatic NEC[21], despite of the similar response to chemotherapy[22, 23]. Still, many HGNECs patients were present with advanced-or late-stage disease, approximately $50 \%$ of the NEC patients with synchronous metastatic disease[5, 24], due to the lack of early symptoms[1], and there is a lack of the optimal treatment HGNECs of the colon and rectum[9].

NECs is quite another malignancy, and it did not benefit from improvement achieved in colorectal carcinoma over the past decade[20,24]. The majority of published data regarding chemotherapy for NECs is derived from patients

Page $13 / 23$ 
with advanced disease. Evidence to support clinical managements for CRNEC is scarce. Due to the rarity of CRNEC and the resulting difficulty to accumulate a large cohort for evaluation, there are few retrospective studies and almost no prospective studies to evaluate its prognostic value of $A C[2]$, resulting in the fact that, due to the histological and clinical resemblance, treatment is more likely in analogy to the much more common SCLC[1, 20], with platinum-based chemotherapy as a cornerstone[9]. In clinical practice, relevant features addressing the optimal management strategy of NECs consists of clinical behavior, mitoses, Ki-67 expression, morphology and so on[5]. Bearing the high relapse rate following radical surgery in mind, platinum-based AC in this setting would be advocated, although it was not supported by sufficient evidence, with no randomized studies ever addressing the effect[1]. The 5-year overall survival (OS) rate varied from 50.1-54.5\% for patients with localized disease, compared to $20.0-29.2 \%$ for patients with regional disease, and the best treatment strategy for patients with nonmetastatic CRNECs remains controversial[8]. Many CRNEC patients recurred rapidly after surgery, implying a role for adjuvant chemotherapy[8,20]. One retrospective study including 132 patients with nonmetastatic CRNECs received operation treatment from the SEER database that has compared AC versus observation for CRNECs, demonstrated that no significant improvement in survival achieved for patients receiving AC compared with those with observation[2], despite of the different regimens selected for chemotherapy. Although it was in correspondence with our findings, this conclusion may be limited due to the small sample of patients, so we enrolled more patients to further confirm it. The present study demonstrated that AC was invalid for improved CSS in patients with nonmetastatic CRNECs regardless of tumor stages. Furthermore, it was notable that the 5year survival (37\%) for patients undergoing both radical surgery and adjuvant chemotherapy remains low, similar to that for the same cohort undergoing surgery alone, at 32\%[9], implying the limited benefit of adjuvant chemotherapy[25]. Differentiation and Ki-67 is able to determine prognostic groups[1, 26]. A multicenter, retrospective study identified higher objective response but poorer survival associated with higher Ki-67 index whose cut-off was 55\% $(P<0.001)[5,23,27]$, coming to the conclusion that NEC with Ki-67 > 55\% may benefit from combination therapies[8]. There are no studies with satisfactory inclusion evaluating the efficacy of adjuvant chemotherapy in patients with CRNECs. Notably, we first assessed the efficacy of AC in nonmetastatic CRNEC of large, small cell patients and precisely diagnosed CRNECs. In our study, only 152 patients (60.56\%) received $A C$, suggesting a poor compliance with the guidelines, one major reason for which is probably attributed to a lack of consensus regarding the role of adjuvant therapy, as no evidence supporting the efficacy of AC existed.

Our study went further to validate the prognostic effects of current $\mathrm{T}$ and $\mathrm{N}$ classification to advocate a more tailored TNM staging system. With the population-based analysis, our result surprisingly demonstrated that both the current $\mathrm{N}$ classification and the $\mathrm{T}$ classification failed to precisely predict the CSS, which was in correspondence with the conclusion from study of Wu Z et al[2]. We suggest that the LNR-based Nr classification is more useful in the CRNECs staging system than the current $\mathrm{N}$ classification system consulted to that of common colorectal carcinoma. LNR-based classification has been proved to be helpful in terms of the breast, stomach, colorectum and so on[10]. Based on the number of positive LNs, the classic N classification can be affected by the LNs yield, which may be influenced by the extent of surgical resection, the following pathological evaluation as well as the characteristics of the tumor. To the best of our knowledge, this is the first study to investigate the role of LNR and the corresponding TNM stage on CSS of CRNEC, and further successfully to advocate the LNR-based classification $(\mathrm{Nr})$ as a better predictor of the CSS. Based on the new advocated $\mathrm{Nr}$ classification, a refined TNrM stage was proposed.

Page 14/23 
By using the PSM method, which has a number of potential advantages over more common matching techniques such as retaining all the samples, the analysis gave a robust conclusion by reducing the estimation bias and improving validity. Despite of the inherit defects, our study has several strengths. With a total of 251 patients who were identified as nonmetastatic CRNEC of large, small cell patients and precisely diagnosed CRNECs, this is the largest study to date that evaluates patients with CRNECs and their treatment outcomes. With these data, we are able to show that AC are not associated with survival in a large, national cohort.

However, our analysis is subject to limitations related to the nature of registry-derived data and the inherent selection bias given by the retrospective study when interpreting the findings. Unmeasured confounders still exist, despite of the effort that a PS-matched technique and a competing-risks model were used to minimize the bias. However, to the best of our knowledge, no randomized study is recruiting for this purpose which the effect of AC in patients with CRNECs is explored. Second, poorly differentiated NECs are characterized by a Ki-67 index $>50-$ $60 \%[8]$. However, the grading of NECs in the SEER database is mainly evaluated by tumor differentiation instead of on Ki-67 index which could not be identified. Third, there is no coding for recurrence as well as the types of chemotherapy in the SEER database, and the effect of AC may be influenced bearing it in mind that chemotherapy was classified even if it was interrupted before completion. So, it is unknown whether shortened or prolonged therapy may affect survival. Forth, there was a significant number of patients had $<12$ lymph nodes examined in our cohort and SEER only collects data for overall survival (OS) rather than disease-specific survival (DFS).

In summary, by using the SEER dataset, our study revises survival based on the LNR and subsequently propose a new evidence-based stage grouping for CRNECs. The new TNrM stage more accurately predict survival of patients with CRNECs than the current AJCC N classification. Furthermore, the present study argues that AC was invalid for improving the survival of patients with nonmetastatic CRNECs following resection. Nevertheless, future work is still needed to externally verify the validation of the revised TNrM stage and the certain role of AC.

\section{Conclusions}

AC seems invalid for improving the survival of patients with nonmetastatic CRNECs following resection. The LNR more accurately predict survival of CRNEC patients than current $\mathrm{N}$ classification. Further clinical studies are required to validate the favorable effect of the current $\mathrm{N}$ classification as well as the revised TNrM staging system.

\section{Declarations}

\section{Funding}

The study was partially supported by a grant from the Key Program of the Jinhua Municipal Science \& Technology Bureau (grant number 2018-3-001d).

The study was partially supported by the Medical Science \& Technology Plan Project of Zhejiang Province (grant number LGF18H160029) and the General Program of Zhejiang Natural Science Foundation (grant number LY19H160020). 
The study was partially supported by a grant from the Key Program of the Jinhua Municipal Science \&

Technology Bureau (grant number 2019-3-013).

\section{Competing Interests}

The authors declare that no competing interests exist.

\section{Availability of Data and Materials}

All the raw data were obtained from the publicly available SEER database with no special permission needed.

\section{Authorship and Contributions}

Design of the study and drafting the manuscript: Xiaoxiao Chen, Hongjuan Zheng. Acquisition of data, collection and review of the literature: Xia Zhang, Wanfen Tang, Shishi Zhou, Xifeng Xu. Critical revision of the manuscript for important content: Jianfei Fu, Qinghua Wang.

\section{Ethical approval and consent to participate section}

This article does not contain any studies with human participants or animals performed by any of the authors.

\section{Consent for publication section}

Not Applicable

\section{References}

Reference

1. Kunz PL, Reidy-Lagunes D, Anthony LB, et al. Consensus guidelines for the management and treatment of neuroendocrine tumors. Pancreas. 2013;42:557-77.

2. Wu Z, Yu D, Zhao S, et al. The efficacy of chemotherapy and operation in patients with colorectal neuroendocrine carcinoma. J Surg Res. 2018;225:54-67.

3. Scherübl H, Jensen RT, Cadiot G, et al. Management of early gastrointestinal neuroendocrine neoplasms. World journal of gastrointestinal endoscopy. 2011;3:133-9.

4. Jernman J, Välimäki MJ, Louhimo J, et al. The novel WHO 2010 classification for gastrointestinal neuroendocrine tumours correlates well with the metastatic potential of rectal neuroendocrine tumours. Neuroendocrinology. 2012;95:317-24.

5. Bruera G, Giuliani A, Romano L. Poorly differentiated neuroendocrine rectal carcinoma with uncommon immune-histochemical features and clinical presentation with a subcutaneous metastasis, treated with first line intensive triplet chemotherapy plus bevacizumab FIr-B/FOx regimen: an experience of multidisciplinary management in clinical practice. 2019; 19: 960.

6. Conte B, George B, Overman M, et al. High-Grade Neuroendocrine Colorectal Carcinomas: A Retrospective Study of 100 Patients. Clin Colorectal Cancer. 2016;15:e1-7.

7. Garcia-Carbonero R, Sorbye H, Baudin E, et al. ENETS Consensus Guidelines for High-Grade Gastroenteropancreatic Neuroendocrine Tumors and Neuroendocrine Carcinomas. Neuroendocrinology. 2016;103:186-94. 
8. Chiominto A, Di Sibio A, Mastropietro S, et al. Adjuvant Chemotherapy Versus Observation Following Resection for Patients With Nonmetastatic Poorly Differentiated Colorectal Neuroendocrine Carcinomas. BMC cancer 2019.

9. Fields AC, Lu P, Vierra BM, et al. Survival in Patients with High-Grade Colorectal Neuroendocrine Carcinomas: The Role of Surgery and Chemotherapy. Ann Surg Oncol. 2019;26:1127-33.

10. Wu L, Chen F, Chen S, Wang L. The Lymph Node Ratio Optimizes Staging in Patients with Small Intestinal Neuroendocrine Tumors. Neuroendocrinology. 2018;107:209-17.

11. Norwood MG, Sutton AJ, West K, et al. Lymph node retrieval in colorectal cancer resection specimens: national standards are achievable, and low numbers are associated with reduced survival. Colorectal disease: the official journal of the Association of Coloproctology of Great Britain Ireland. 2010;12:304-9.

12. Belt EJ, te Velde EA, Krijgsman O, et al. High lymph node yield is related to microsatellite instability in colon cancer. Ann Surg Oncol. 2012;19:1222-30.

13. Etzioni D, Spencer M. Nodal harvest: surgeon or pathologist? Dis Colon Rectum. 2008;51:366-7. author reply 8.

14. Giuliani A, Romano L, Coletti G, et al. Lymphangiomatosis of the ileum with perforation: A case report and review of the literature. Annals of medicine surgery (2012). 2019;41:6-10.

15. Dasari A, Shen C, Halperin D, et al. Trends in the Incidence, Prevalence, and Survival Outcomes in Patients With Neuroendocrine Tumors in the United States. JAMA oncology. 2017;3:1335-42.

16. Minocha V, Shuja S, Ali R, Eid E. Large cell neuroendocrine carcinoma of the rectum presenting with extensive metastatic disease. Case Rep Oncol Med 2014; 2014: 386379.

17. Brenner B, Shah MA, Gonen M, et al. Small-cell carcinoma of the gastrointestinal tract: a retrospective study of 64 cases. British journal of cancer. 2004;90:1720-6.

18. Walter T, Tougeron D, Baudin E, et al. Poorly differentiated gastro-entero-pancreatic neuroendocrine carcinomas: Are they really heterogeneous? Insights from the FFCD-GTE national cohort. Eur J Cancer. 2017;79:158-65.

19. Kyriakopoulos G, Mavroeidi V, Chatzellis E, et al. Histopathological, immunohistochemical, genetic and molecular markers of neuroendocrine neoplasms. Annals of translational medicine. 2018;6:252.

20. Shafqat H, Ali S, Salhab M, Olszewski AJ. Survival of Patients With Neuroendocrine Carcinoma of the Colon and Rectum: A Population-Based Analysis. Diseases of the Colon Rectum. 2015;58:294.

21. La Rosa S, Sessa F. High-grade poorly differentiated neuroendocrine carcinomas of the gastroenteropancreatic system: from morphology to proliferation and back. Endocrine pathology. 2014;25:193-8.

22. Heetfeld $\mathrm{M}$, Chougnet $\mathrm{CN}$, Olsen $\mathrm{IH}$, et al. Characteristics and treatment of patients with $\mathrm{G} 3$ gastroenteropancreatic neuroendocrine neoplasms. Endocrine-related Cancer. 2015;22:657-64.

23. Sorbye H, Welin S, Langer SW, et al. Predictive and prognostic factors for treatment and survival in 305 patients with advanced gastrointestinal neuroendocrine carcinoma (WHO G3): the NORDIC NEC study. Annals of oncology: official journal of the European Society for Medical Oncology. 2013;24:152-60.

24. Shafqat H, Ali S, Salhab M, Olszewski AJ. Survival of patients with neuroendocrine carcinoma of the colon and rectum: a population-based analysis. Dis Colon Rectum. 2015;58:294-303. 
25. Balasubramanyam S, O'Donnell BP, Musher BL, et al. Evaluating Treatment Patterns for Small Cell Carcinoma of the Colon Using the National Cancer Database (NCDB). Journal of gastrointestinal cancer. 2019;50:24453.

26. Vélayoudom-Céphise FL, Duvillard P, Foucan L, et al. Are G3 ENETS neuroendocrine neoplasms heterogeneous? Endocrine-related Cancer. 2013;20:649-57.

27. Milione M, Maisonneuve P, Spada F, et al. The Clinicopathologic Heterogeneity of Grade 3

Gastroenteropancreatic Neuroendocrine Neoplasms: Morphological Differentiation and Proliferation Identify Different Prognostic Categories. Neuroendocrinology. 2017;104:85-93.

\section{Figures}

\section{Inclusion and exclusion criteria in SEER}

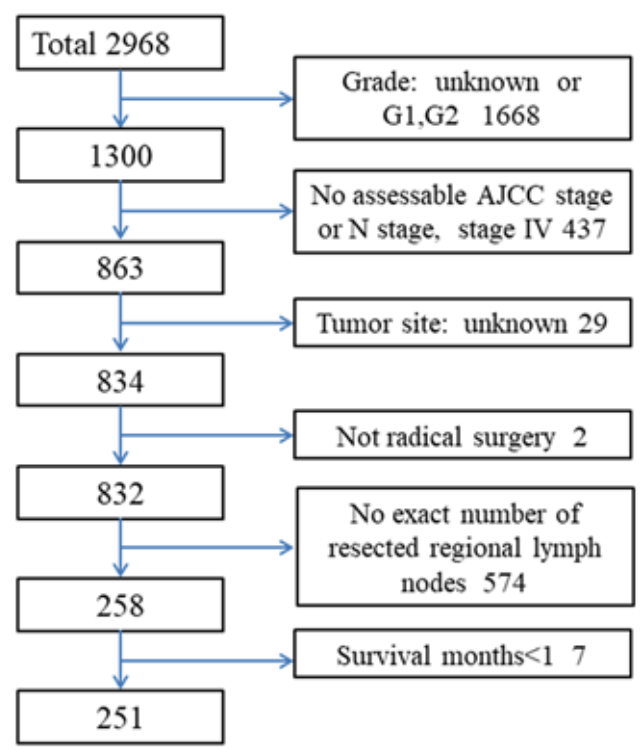

Selection in SEER

Sex $=$ male, female

Year of diagnosis=1992-2016

Site record $=$ colon, rectum

Diagnostic

confirmation $=$ microscopically confirmed

Histology $=8013 / 3,8041 / 3,8246 / 3$

Survival months !=unknown

Sequence $=$ one primary only

Age at diagnosis $=20-80$

Marital status $=$ single, married, divorced

Race recode $=$ white, black, other

\section{Figure 1}

Inclusion and exclusion criteria in SEER. 
A

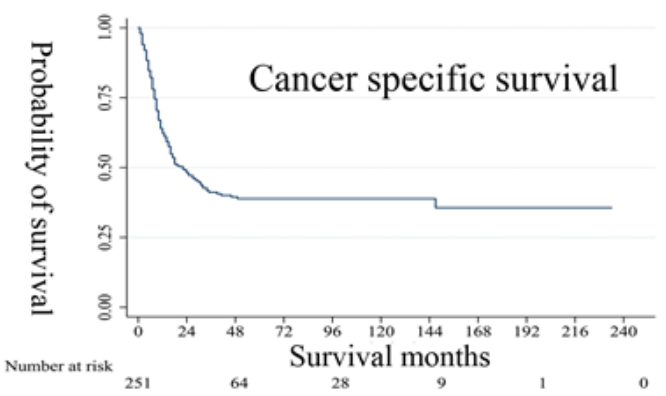

$\mathrm{C}$

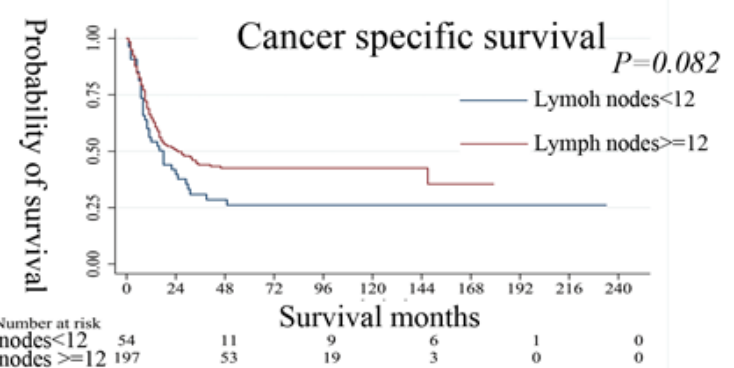

B

Restrict cubic spline method for regional lymph nodes

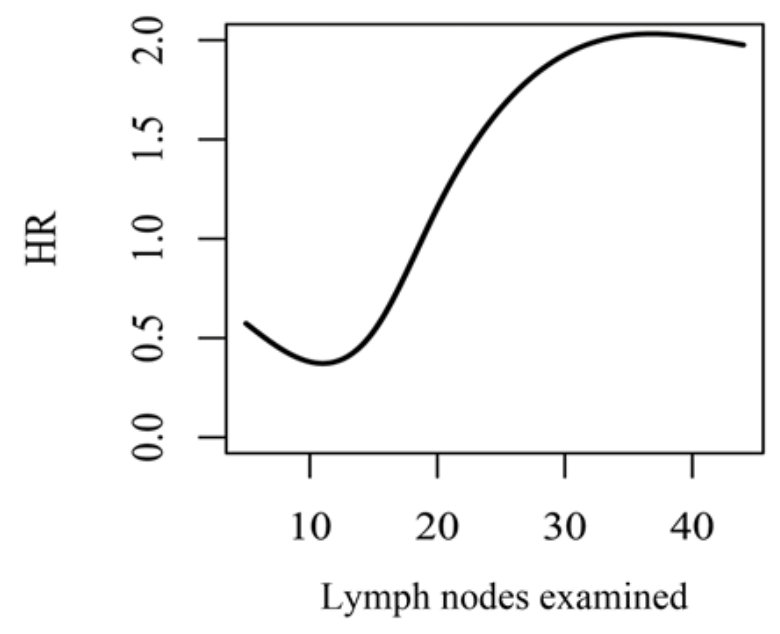

\section{Figure 2}

poor prognosis of NEC and influence of $\mathrm{N}$ classification.

A

Restrict cubic spline method for age

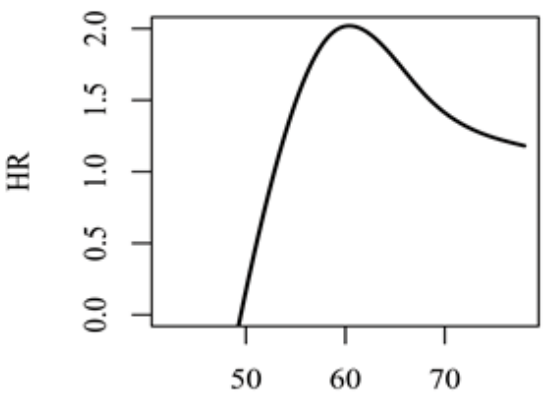

age

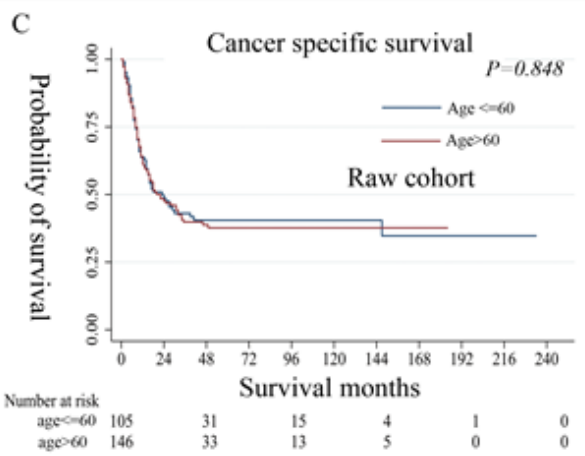

B

Cumulative incidence curves by gary test method

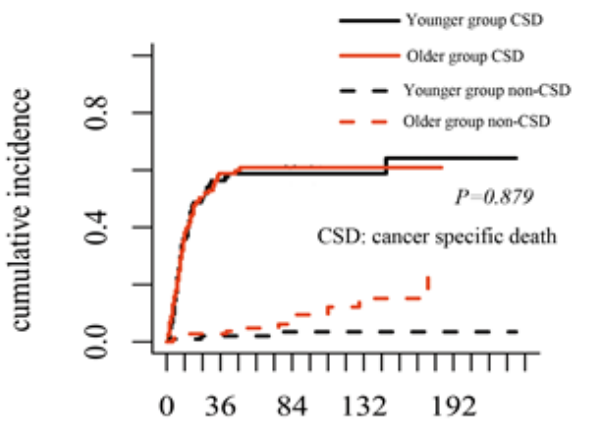

Follow up time (months)

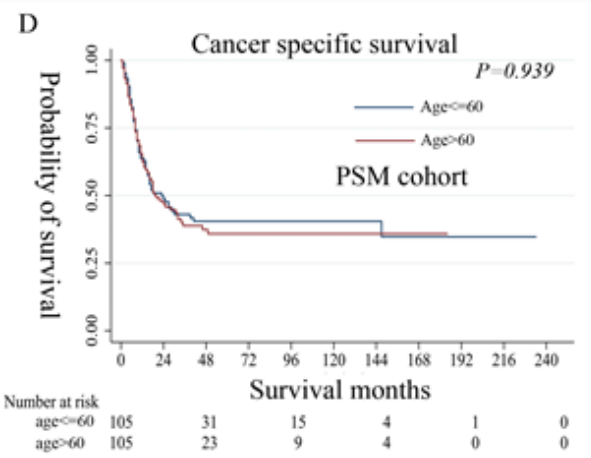

Figure 3 
Influence of age on CSS.

A Cumulative incidence curves by gray test method

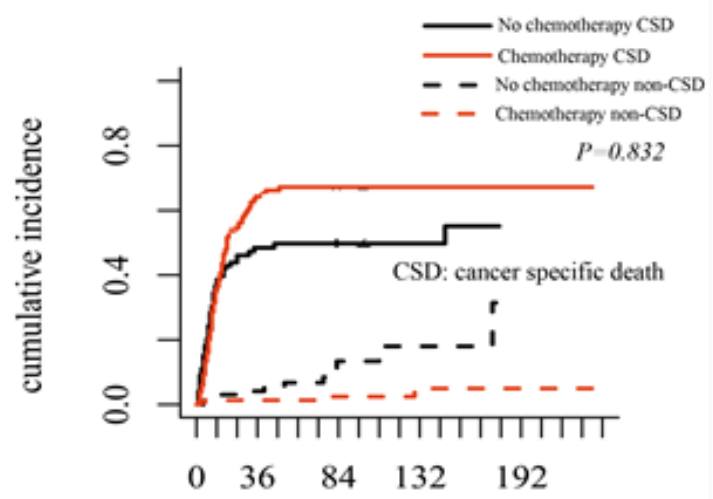

B
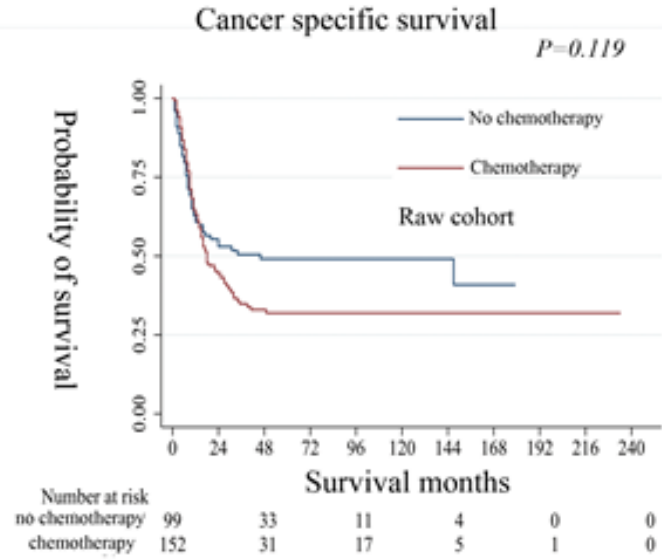

Follow up time (months)

C

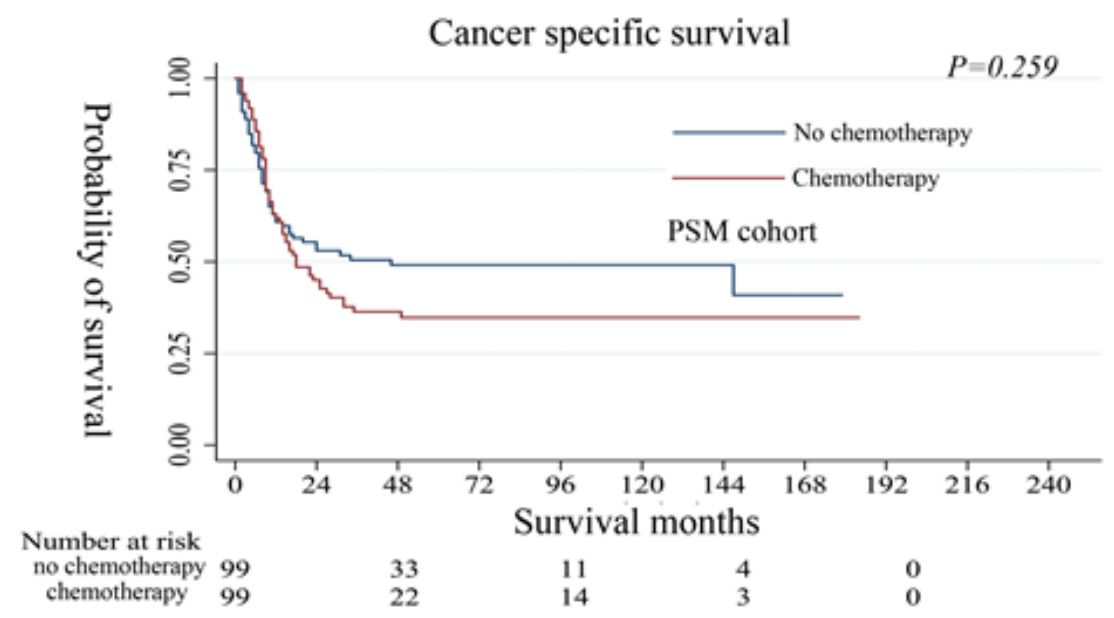

Figure 4

Influence of chemotherapy on CSS. 
A

Cancer specific survival

$P=0.020$

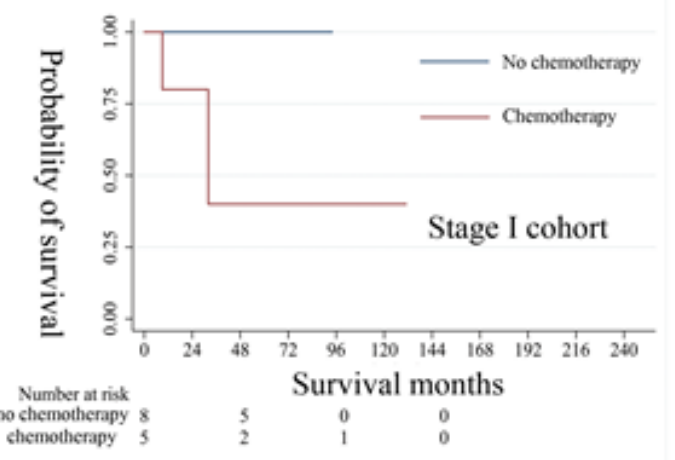

B

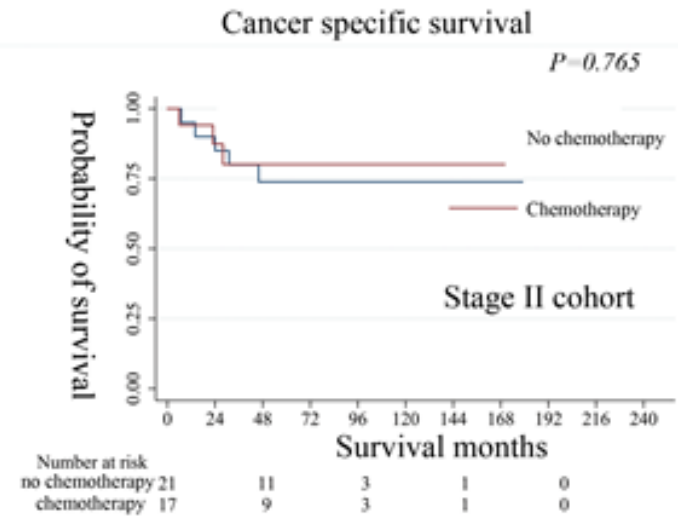

C

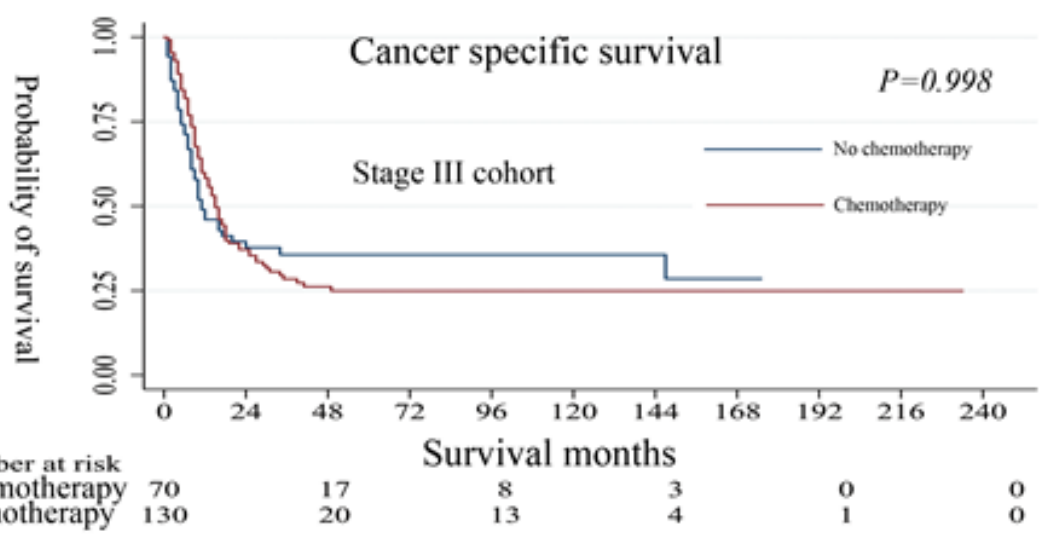

Figure 5

influenc of chemotherapy on different stage. 
A

Current TNM staging system AJCC-8

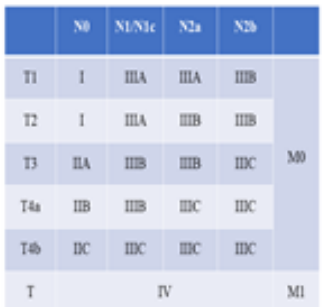

Version 2.02019

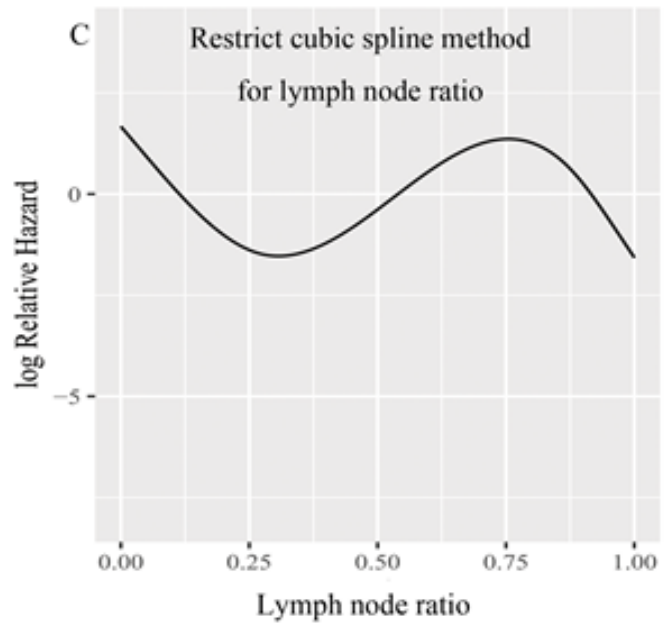

B

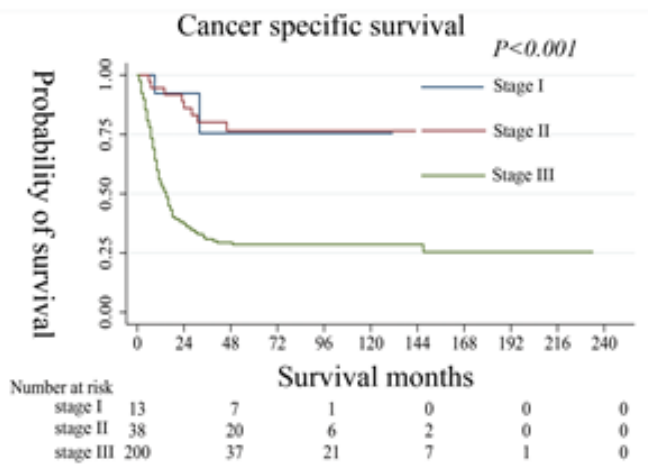

D

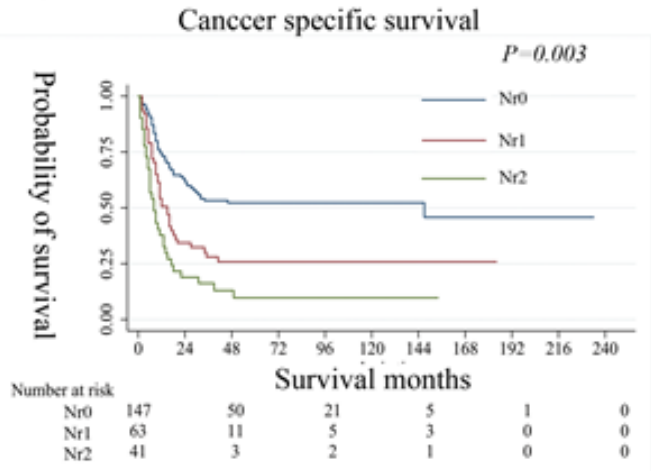

\section{Figure 6}

predictive value of current stage and revised $\mathrm{Nr}$ stage. 
A

Revised TNM staging system

\begin{tabular}{|c|c|c|c|c|}
\hline & Nr0 & Nr1 & Nr2 & \\
\hline T1 & I & II & III & \\
T2 & I & II & III & \\
\hline T3 & II & II & III & M0 \\
\hline T4 & II & III & III & \\
\hline T & & IV & & Ml \\
\hline
\end{tabular}

B

Cancer specific survival

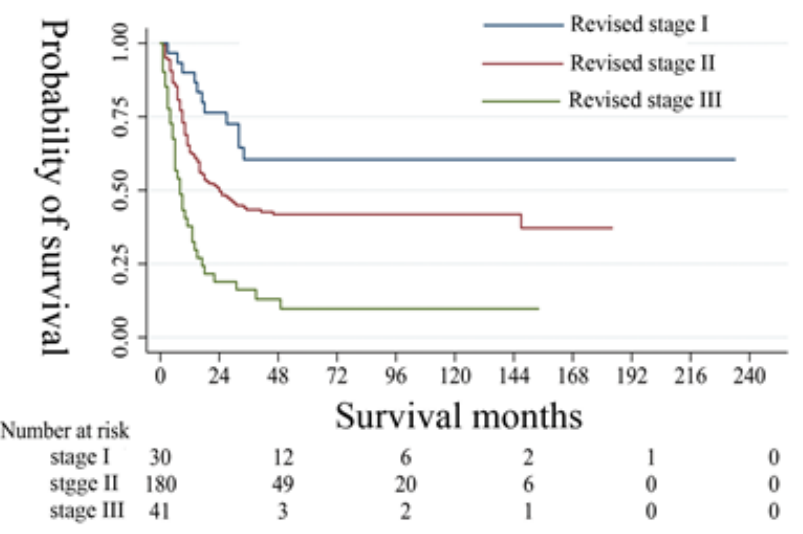

Figure 7

Revised stage and its predictive value. 